mit dürren Worten *) als etwas Bewiesenes hingestellt und dadurch den Irrthum verbreitet hat, dessen Berichtigung wir $\mathrm{W}$ is bar verdanken?

\title{
Ueber die kritischen Temperaturen von
}

Flüssigkeitsgemischen ;

\author{
von G. C. Schmidt.
}

(Eingelaufen am 6. August 1891.)

Untersuchungen über die kritischen Constanten von Flüssigkeiten sind bisher verhältnifsmäfsig nur wenig angestellt worden. Die beträchtlichen Schwierigkeiten, welche namentlich mit der Bestimmung des kritischen Druckes und Volums verbunden sind, sind wohl die Ursache gewesen, dafs dieser Theil der physikalischen Chemie vernachlässigt worden ist, obwohl die Kenntnifs des kritischen Constanten auf viele Gebiete der Stöchiometrie ein neues Licht zu werfen verspricht. Dazu kommt, dafs die Arbeit wegen der grofsen Abweichungen, die man mit verschiedenen Präparaten erhält, keine rechte Befriedigung gewährt. Selbst Körper, die in beliebiger Menge anscheinend absolut rein hergestellt werden können, weichen in ihren kritischen Constanten stark von einander ab. Beispiele hierfür werde ich im Laufe der folgenden Abhandlung mehrfach anführen. Trotzdem hoffe ich binnen Kurzem eine Reihe zuverlässiger Messungen kritischer Constanten veröffentlichen zu können.

Die folgende Arbeit verdankt ihre Entstehung den Ab-

*) Diese Annalen 101, 48. 


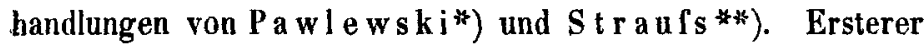
hat die von ihm gefundenen Resultate folgendermafsen zusammengefafst :

I. Die kritischen Temperaturen $\vartheta$ homologer Verbindungen unterscheiden sich von ihren Siedetemperaturen $t$ um eine constante Differenz und können durch den einfachen Ausdruck $\vartheta=1+$ Const. bezeichnet werden.

II. Isomere Ester besitzen gleiche oder sehr ähnliche kritische Temperaturen. Bei anderen isomeren Verbindungen, z. B. bei den Alkoholen, ist dies indefs, wie sich auch aus dem vorhergehenden Satze unmittelbar ergiebt, nicht der Fall.

III. Die kritische Temperatur $9 \mathrm{~m}$ einer Mischung zweier Flüssigkeiten kann ausgedrückt werden durch die Formel :

$$
\vartheta \mathrm{m}=\frac{\mathrm{n} \vartheta+(100-\mathrm{n}) \vartheta^{\prime}}{100}
$$

in welcher $\mathrm{n}$ und $100-\mathrm{n}$ die procentischen Mischungsverhältnisse der Bestandtheile, $\vartheta$ und $\vartheta^{\prime}$ die kritischen Temperaturen derselben bedeuten.

Obgleich die von $\mathrm{Pawlewski}$ bestimmten kritischen Temperaturen, welche sich auf die Ester der homologen Reihe der Fettsäuren beziehen, seine letzten beiden Sätze gut bestätigen, haben 0 stwald $* * *$ ) und Bartolif) Einwendungen gegen dieselben erhoben, welche sicherlich begründet sind. Da nämlich das erste Gesetz, wenn man es auf irgend einen anderen Druck als den zufälligen Druck von $760 \mathrm{~mm}$ überträgt, nothwendig die Geltung des als falsch erkannten D a Ito $n$ 'schen Gesetzes, dafs gleichen Aenderungen der Siede-

*) Ber. d. deutsch. chem. Ges. I5, 460 u. 2460 .

**) Beibl. 6, 282.

***) O stwald, Lehrbuch Bd. 1, pag. 308 .

†) Beibl. 9, 721. 
temperatur gleiche Aenderungen des Drucks entsprechen, voraussetzt, so kann der Regel von $P$ a wlewski nur eine annähernde Geltung zugeschrieben werden. Die Kolumnen, welche 0 stwald der Tabelle $P$ awlewski's hinzugefügt hat, beweisen, dafs $\vartheta-t_{130}, \vartheta-t_{76}, \vartheta-t_{20}$ (wo $\vartheta$ die kritische Temperaturen $t_{130}, t_{76}, t_{20}$ die von Schumann*) bestimmten Siedepunkte unter 130, 76 resp. $20 \mathrm{~cm}$ Druck bedeuten) keineswegs constant sind, sondern Abweichungen bis $10^{\circ} \mathrm{C}$. zeigen.

Ahgesehen von den Zahlen von Pawlewski liegen noch weitere Messungen von verschiedenen Beobachtern vor, welche eine Prüfung der ersten beiden Sätze erlauben. In der folgenden Tabelle habe ich die kritischen Temperaturen der homologen Reihe der Fettalkohole zusammengestellt :

\begin{tabular}{|c|c|c|c|}
\hline Substanzen & Krit. Temp. & $\theta-t_{76}$ & Beobachter **) \\
\hline Methylalkohol & $\begin{array}{l}232,7 \\
240,0 \\
233,0 \\
242,0\end{array}$ & $\begin{array}{l}166,7 \\
175,0 \\
170,0 \\
177,3\end{array}$ & $\begin{array}{l}\text { Hannay. } \\
\text { Ramsay \& Young. } \\
\text { Nadejdine. } \\
\text { Sohmidt ***). }\end{array}$ \\
\hline Aethylalkohol & $\begin{array}{l}234,3 \\
243,6 \\
234,6 \\
235,5 \\
240,6\end{array}$ & $\begin{array}{l}155,3 \\
164,6 \\
155,6 \\
156,5 \\
161,6\end{array}$ & $\begin{array}{l}\text { Sajotschewski. } \\
\text { Ramsay \& Young. } \\
\text { Hannay \& Hogarth.. } \\
\text { Hannay. } \\
\text { Straufs. }\end{array}$ \\
\hline Propylalkohol & $\begin{array}{l}263,7 \\
261,0 \\
256,0 \\
270,5\end{array}$ & $\begin{array}{l}166,7 \\
164,0 \\
159,0 \\
173,7\end{array}$ & $\begin{array}{l}\text { Ramsay \& Young. } \\
\text { de Heell. } \\
\text { Nadejdine } \\
\text { Schmidt ***). }\end{array}$ \\
\hline Butylalkohol & $\begin{array}{l}287,1 \\
270,5\end{array}$ & $\begin{array}{l}170,1 \\
153,5\end{array}$ & $\begin{array}{l}\text { Pawlewski. } \\
\text { de Heen. }\end{array}$ \\
\hline Isopropylalkohol & $\begin{array}{l}234,6 \\
238,0\end{array}$ & $\begin{array}{l}151,6 \\
155,0\end{array}$ & $\begin{array}{l}\text { Nadejdine. } \\
\text { de Heen. }\end{array}$ \\
\hline Isobutylalkohol & 265,0 & 167,0 & Nadejdine. \\
\hline 1soamylalkohol & 306,6 & 176,6 & Pawlewski. \\
\hline
\end{tabular}

*) Wied. Ann. 12, 40, 1881.

**) Eine vollständige Zusammenstellung der bisher ermittelten kritischen Daten von Flüssigkeiten findet sich in Zeitschr. f. physik. Chemie Bd. \&, S. 601 von B. Heilborn. Allda befinden sich auch die Litteraturnachweise zu der obenstehenden Tabelle.

**) Diese Abhandlung S. 276 u. 279. 
Wenn auch die Beobachtungen verschiedener Forscher aufserordentlich von einander abweichen, kann doch mit voller Sicherheit der Schluls gezogen werden, dafs der erste Satz von $\mathrm{Pawlewski}$ für die homologe Reihe der Fettalkohole nicht richtig ist; die kritische Temperatur des Methylalkohols ist wahrscheinlich höher als die des Aethylalkohols, obwohl der Siedepunkt des Methylalkohols unter Atmosphärendruck $13^{\circ} \mathrm{C}$. tiefer liegt. Ein ähnliches Bild gewinnt man durch Zusammenstellung der kritischen Temperaturen anderer homologer Reihen, z. B. der Aether und Amine. Ich führe die Rechnung nicht durch, da diese Körper meistens nur von einem Beobachter und an einem Präparat untersucht worden sind und daher die Ergebnisse noch weniger zuverlässig sind, als die oben mitgetheilten.

Auch die anderen Regeln von Pawlewski sind nicht ohne Widerspruch geblieben; von verschiedener Seite sind experimentelle Belege dafür erbracht worden, dafs der dritte Satz, nach dem die kritische Temperatur einer Mischung sich nach der Formel

$$
\vartheta \mathrm{m}=\frac{\alpha \mathrm{T}_{1}+\beta \mathrm{T}_{2}}{\alpha+\beta}
$$

berechnen lasse, nicht in allen Fällen zutreffe.

So hat Ansdell*) die kritischen Temperaturen von Gemischen der Gase Chlorwasserstoff und Kohlensãure untersucht, wobei sich ergab, dafs die berechneten Werthe stets kleiner ausfielen, als die nach der Formel von Paw lewski berechneten.

Die folgende Tabelle, welche ich aus den Beobachtungen von Ansdell berechnet habe, giebt einen Ueberblick über die Gröfse der Abweichungen :

*) Proc. Roy. Suc. a 4, 113. 1882. Beibl. 7, 257. 


\begin{tabular}{c|c|c|c}
\hline \hline$\%$ Kohlensäure & $\vartheta$ & $\vartheta$ berechnet & Differenz \\
\hline $\mathrm{CO}_{\mathbf{8}}$ & $31,1^{\circ} \mathrm{C}$. & - & - \\
17,18 & $47,2^{\circ} \mathrm{C}$. & $47,8^{\circ} \mathrm{C}$. & $-0,6$ \\
19,37 & $45,5^{\circ} \mathrm{C}$. & $47,3^{0} \mathrm{C}$. & $-1,8$ \\
25,48 & $45,1^{0} \mathrm{C}$. & $46,1^{\circ} \mathrm{C}$. & $-1,0$ \\
42,44 & $39,5^{\circ} \mathrm{C}$. & $42,7^{\circ} \mathrm{C}$. & $-3,2$ \\
45,67 & $38,0^{\circ} \mathrm{C}$. & $42,0^{\circ} \mathrm{C}$. & $-4,0$ \\
74,18 & $33,5^{\circ} \mathrm{C}$. & $36,3^{\circ} \mathrm{C}$. & $-2,8$ \\
82,14 & $32,4^{\circ} \mathrm{C}$. & $34,7^{\circ} \mathrm{C}$. & $-2,3$
\end{tabular}

Die kritische Temperatur des Chlorwasserstoffs war $51,25 " \mathrm{C}$.

Zu einem ähnlichen Ergebnifs wie Ansdell gelangte v. d. Wa als*) bei der Untersuchung eines Gemenges von $7 \mathrm{Vol} . \mathrm{CO}_{2}$ und $3 \mathrm{Vol}$. HCl. Die kritische Temperatur ergab sich zu $31,6^{\circ} \mathrm{C}$, während sie nach $\mathrm{Pawlewski} 36,2^{\circ} \mathrm{C}$. sein sollte; der beobachtete Werth ist also ebenfalls kleiner, als der nach der Formel von Pawlewski berechnete.

Obwohl diese Untersuchungen zu beweisen scheinen, dafs die kritischen Temperaturen gewöhnlicher Gasgemische nicht mit ausreichender Genauigkeit durch die Formel von Pawlewski dargestellt werden, so kann diese Frage noch nicht als endgültig entschieden betrachtet werden. Einerseits sind die experimentellen Daten noch nicht zahireich genug, andererseits sind die Versuche stets mit Kohlensäure angestellt worden, einem Gase, das sich nach den Untersuchungen von Dew a ${ }^{* *}$ ) bei hohen Drucken und hohen Temperaturen mit anderen Körpern chemisch verbindet. Ob hierdurch die oben

*) Van der Waals : Continuität des gasförmigen und flüssigen Zustandes. Leipzig 1881, S. 142.

**) Nach den Untersuchungen von Dewar bildet die Kohlensüure bei hohen Drucken und Temperaturen mit Schwefelkohlenstoff, Chloroform, Benzol, Aether, Phosphorchlorür u. s. w. unbeständige chemische Verbindungen, die sich bilden und zersetzen, je nach dem Druck und der Temperatur. Proc. Roy. Soc. Lond. 80, 338. 1880. Beibl. 4, 771 . 
erwähnten Abweichungen von der Paw lew ski'schen Formel erklärt werden, mufs vorläufig dahingestellt bleiben.

Auch bei Flüssigkeitsgemischen haben sich beträchtliche Differenzen zwischen den beobachteten und den nach dem P awle ws ki'schen Gesetz berechneten Zahlen ergeben. So fand B. Galitzine*) die kritischen Temperaturen von Gemischen von Schwefelkohlenstoff und Aethyläther erheblich kleiner, als es die P a wlew sk i'sche Formel verlangt. Gemische von Aethyläther und Aceton zeigten ein ähnliches Verhalten, wenn auch der Unterschied zwischen beobachteten und berechneten Werthen nicht bedeutend war, nämlich im Maximo $2,6^{0} \mathrm{C}$.

Ein Gemisch von gleichen Gewichtstheilen Aethyläther und Benzol ist von Rams a ${ }^{* *)}$ untersucht worden. Die kritischen Temperaturen der Bestandttheile sind 195,5 resp291,5, diejenige des Gemisches $240,7^{\circ} \mathrm{C}$, während sie nach Pawlewski $243,6^{0} \mathrm{C}$. sein sollte. Der beobachtete Werth ist also wiederum kleiner, als der berechnete.

Dagegen zeigen die Untersuchungen von 0 . Strau fs ${ }^{*} * * *$ ) über Gemische von Alkohol und Aether, dafs die Formel von Pawlewski für diese Flüssigkeiten als richtig betrachtet werden darf.

Fassen wir die Ergebnisse der oben erwähnten Untersuchungen zusammen, so scheinen sie für die Formel von Pa w lewski nicht günstig zu sein; die beobachteten Werthe sind in allen Fällen sowohl bei Gas als auch bei Flüssigkeitsgemischen niedriger als die berechneten.

Entgültig entschieden ist die Frage jedoch noch nicht;

*) Wied. Annalen 41, 623.

**) Proc. Roy. Soc. 81, 194. - Beibl. 5, 422.

*** O. Straufs, Journ. d. russ. phys.-chem. Ges. 18, 207. Beibl. G, 282 . 1882 . 
denn einerseits erstrecken sich die Versuche über zu wenige Körper, andrerseits lassen sich gegen eine Reihe der erhaltenen Resultate Einwendungen erheben, auf die vielleicht die Abweichungen von der Paw lew ski'schen Regel zurückgeführt werden können. Bei den Versuchen Galitzin e's über die Gemische von Schwefelkohlenstoff und Aether hat sich stets Schwefelwasserstoff gebildet; es hat also eine tiefgreifende Zersetzung und Umlagerung der Atome stattgefunden, so dafs diese Versuche zur Entscheidung der Frage, ob die Pawlewski'sche Formel die kritischen Temperaturen von Flüssigkeitsgemischen mit ausreichender Genauigkeit wiedergiebt oder nicht, nicht herangezogen werden dürfen. Die Differenzen bei den von Galitzine untersuchten Gemischen von Aethyläther und Aceton, welche im Maximum $2,6^{\circ} \mathrm{C}$. betrugen, erklären sich leicht dadurch, dafs die Polymerisation des Acetons durch Zusatz von Aether verlangsamt wurde, so dafs die kritische Temperatur nicht so hoch steigen konnte als bei dem unvermischten Aceton. Je mehr Aether in der Mischung enthalten war, desto gröfser mufsten in Folge dessen die Differenzen zwischen den beobachteten und berechneten Werthen werden, wie es auch thatsächlich der Fall war.

Um die Frage zu entscheiden, ob die Formel von P a wlewski für Flüssigkeitsgemische zulässig ist oder nicht, bedarf es noch weiterer Untersuchungen. Solche Versuche sind von mir angestellt worden. Mein Bestreben ist es dabei gewesen, Mischungen von Flüssigkeiten zu untersuchen, welche die gröfsten Verschiedenheiten in Bezug auf alle ihre physikalischen und chemischen Eigenschaften zeigen, da zu erwarten war, dafs die Formel von P a wlew ski, wenn überhaupt, in diesen Fällen am ersten versagen würde.

In den Versuchen über die kritischen Temperaturen kam ein Luftbad zur Verwendung, das genau nach der Beschrei- 
bung von B. Galitzine *) angefertigt war. Dasselbe bestand aus vier in einander passenden Kästen aus Eisenblech, welche sich an keiner Stelle berührten. In den entgegengesetzten Wänden derselben waren Glasscheiben zur Beobachtung des Versuchs angebracht. Die Abstände zwischen den Wänden der einzelnen Kästen waren mit Asbest ausgefüllt, um einem allzujähen Temperaturabfall vorzubeugen. Das Versuchsrohr aus dickwandigem Glas wurde zuerst ganz mit der zu untersuchenden Flüssigkeit gefüllt; nachdem darauf durch Auskochen die Luft aus dem Rohr vertrieben und die nöthige Menge Flüssigkeit entfernt worden war, wurde das Rohr zu einer dickwandigen Capillare zugeschmolzen. Das Röhrchen wurde mit dem Thermometer in dem innersten Kasten aufgehängt und das Luftbad durch 21 bis $27 \mathrm{Bun-}$ sen'sche Brenner erhitzt. Notirt wurde sowohl die Temperatur, bei der die Flüssigkeit vollständig verschwand, als auch die Temperatur im Moment der Nebelbildung.

Die so angestellten Versuche nahmen sehr viel Zeit in Anspruch: ferner wurde durch die grofse Hitze, welche bei der Erwärmung des Luftbades entwickelt werden mufste, die Temperatur im Laboratorium in den heifsen Sommermonaten so unerträglich, dafs ich versuchte, die kritischen Temperaturen auf einfachere Weise zu bestimmen. Das Verfahren, welches zur Anwendung kam, und das hinsichtlich der Annehmlichkeit und Schnelligkeit der Ausführung nichts zu wünschen übrig liefs, war folgendes. Ein Becherglas, dessen Höhe $250 \mathrm{~mm}$ und dessen Durchmesser ca. $60 \mathrm{~mm}$ betrug, wurde zur Hälfte mit Paraffin gefültt. Ein kleines Röhrchen, welches die zu untersuchende Flüssigkeit enthielt, wurde an das Thermometer befestigt und so in das Paraffinbad gehängt,

*) a. a. O. S. 605 . Auf S. 620 befindet sich auch eine Figur des Lufthades. 
dafs es vollständig von Paraffin umgeben war. Anfangs wurde schnell erwärmt, bis die Temperatur einige Grade unter der kritischen sich befand, darauf langsam unter fortwährendem Rühren. Notirt wurde wiederum sowohl die Temperatur, bei der die Flüssigkeit vollständig verschwand, als auch die Temperatur im Moment des Wiedererscheinens des Meniscus.

Die Methode giebt ausgezeichnete Resultate, nur hat sie den Uebelstand, dafs sie blos bis circa $260^{\circ}$ anwendbar ist. Bei höheren Temperaturen schwärzt sich das Paraflin so schnell, dafs man nach kurzer Zeit nicht mehr die Temperatur genau beobachten kann. Ferner kühlt sich das Paraflin bei diesen hohen Temperaturen so rasch ab, dafs es nur bei ganz aufserordentlicher Sorgfalt im Reguliren der Gasflamme möglich ist, ubereinstimmende Resultate zu erhalten. Versuche, welche ich mit einer Mischung von Kali- und Natronsalpeter anstellte, führten zu keinen befriedigenden Resultaten. Selbstverständlich wurde bei Anstellung dieser Versuche das Gesicht gegen Explosionsgefahr durch eine Glasscheibe geschützt.

Die benutzten Thermometer waren sogenannte Anschützsche, von Gerhardt in Bonn bezogen, in $1 / 5$ getheilt. Da dieselben stets ihrer ganzen Länge nach im Luft- resp. Paraffinbad sich befanden, so brauchte die etwas unsichere Correctur für den herausragenden Faden nicht angebracht zu werden. Am Schlufs der Untersuchung sind dieselben in der physik.technischen Reichsanstalt geprüft worden.

In Betreff der angewandten Substanzen bemerke ich schliefslich, dafs alle Präparate einer sorgfältigen Reinigung unterzogen worden sind, die im speciellen Theil genauer beschrieben werden soll. Zum Fractioniren wurde ein Linneman n'sches Destillationsrohr benutzt. Die gröfste Sorgfalt wurde angewandt, um zu verhindern, dafs die Körper Feuch- 
tigkeit aus der Luft anzogen, da die kritischen Temperaturen durch geringe Mengen von Wasser beträchtlich erhöht werden.

1. Gemische von Methylalkohol und Aether.

Der zu den Versuchen benutzte Methylalkohol (acetonfrei) war von Trommsdorff bezogen. Nachdem er melirere Monate lang über gebranntem Kalk gestanden, wurde er der fractionirten Destillation unterworfen. Der Siedepunkt ergab sich $\mathrm{zu} 64,7$ bis $64,8^{\circ}$ beim Barometerstand $761,8 \mathrm{~mm}$. Um jede Spur Feuchtigkeit, welche der Alkohol beim Destilliren angezogen haben könnte, zu entfernen, wurde in das Destillat ein Stück von chemisch reinem gebrannten Kalk geworfen und die Flasche dann wohl verschlossen.

Röhre I.

$\begin{array}{rllll}\vartheta=242,6 & 242,4 & 242,6 & 242,6 & 242,2 \\ 242,6 & 241,2 & 242,6 & 242,0 & 241,6 \\ 241,8 & 242,7 & 241,4 & & \end{array}$

Röhre II.

Mittel $242,2^{0}$.

\begin{tabular}{|c|c|c|c|}
\hline $9=242,2$ & 243,2 & 242,6 & 243,2 \\
\hline 242,6 & 242,4 & 242,9 & 242,3 \\
\hline 242,1 & 242,6 & 242,4 & \\
\hline
\end{tabular}

Die kritische Temperatur des Methylalkohols ist also $\mathbf{2 4 2 , 4 ^ { 0 }}$. Dasselbe Präparat wurde nochmals der fractionirten Destillation unterworfen und die kritische Temperatur bestimmt. Siedepunkt $64,7^{\circ}$ beim Barometerstand $760,1 \mathrm{~mm}$.

Röhre I.

$$
\begin{array}{rrrrr}
\vartheta=242,4 & 241,0 & 241,4 & 242,0 & 241,4 \\
241,8 & 241,0 & 242,2 & 241,2 & 242,0 \\
242,4 & 241,2 & \multicolumn{2}{c}{\text { Mittel }} & 241,7^{\circ} .
\end{array}
$$

Röbre II.

$$
\begin{array}{rlllr}
\vartheta=242,2 & 241,7 & 242,6 & 241,9 & 242,6 \\
242,6 & 242,4 & 241,8 & 242,8 & 241,4 \\
242,2 & 241,6 . & \multicolumn{2}{c}{\text { Mittel }} & 242,1^{\circ} .
\end{array}
$$


Das Mittel aus allen Ablesungen ist 241,90.

Ein anderes Präparat, von K a hlba u m bezogen, gab folgende kritische Temperaturen.

Röhre I.

$$
\begin{aligned}
& \vartheta=241,9 \quad 240,7 \quad 241,4 \quad 240,8 \quad 241,5 \\
& 241,0 \quad 241,7 \quad 240,1 \quad 240,8 \quad 240,4 \\
& \text { Mittel } 241,0^{\circ} \text {. }
\end{aligned}
$$

Röhre II.

$$
\begin{array}{rllll}
\vartheta=243,4 & 242,6 & 243,4 & 242,5 & 243,6 \\
242,7 & 243,6 & 242,8 & 243,4 & 242,9 \\
243,2 & 242,8 & 243,6 & 242,9 . \\
\multicolumn{4}{c}{\text { Mittel }} & 243,1^{0} .
\end{array}
$$

Die Versuche mit Röhre II waren 2 Tage später angestellt, als die mit Röhre I. Obgleich das Präparat in einer gut verschlossenen Flasche aufbewahrt worden war, hat es doch wahrscheinlich so viel Feuchtigkeit angezogen, dafs die kritische Temperatur um $2^{0}$ erhöht worden ist.

Frühere Versuche ergaben für die kritische Temperatur des Methylalkohols :

$$
\begin{aligned}
& \vartheta=232,76 \pm 0,26 \quad \text { Hannay*) } \\
& 239,9-240,0 \quad \text { Ramsay } u_{0}, Y_{0} \text { ung } \mathrm{g}^{* *} \text { ) } \\
& \text { 233,0 Nadejdine }{ }^{* * *} \text { ) } \\
& \text { 263.0 de Heent). }
\end{aligned}
$$

Aether.

Das benutzte Präparat stammte von Ka hlba um. Nach dem Trocknen mittelst Natrium wurde es fractionirt. Siedepunkt $34,9^{0}$ beim Barometerstand $765,7 \mathrm{~mm}$.

*) Transact. of, Roy. Soc. $\mathbf{3 2}, 294.1882$.

**) Daselbst $18,321.1887$.

***) Journ. d. russ. phys.-chem. Ges. 14, 510. 1882. BeibI. 7, 676. 1883.

†) Recherches touchant la physique comparée et la Théorie des liquides. Paris 1888. Part. expér. p. 102. 
Von Zeit zu Zeit wurde in das Destillat eine blanke Scheibe Natrium geworfen, um jede Spur Feuchtigkeit, welche der Aether mit grofser Begierde anzieht, zu entfernen.

Röhre I.

$\begin{array}{rlllll}\vartheta=192,9 & 193,9 & 192,9 & 194,0 & 193,5 & 193,6 \\ 193,2 & 193,5 & 192,7 & 193,4 & 193,5 & 194,1 \\ 193,2 & 193,8 & 193,2 & & & \end{array}$

Mittel $193,4^{0}$.

Röhre II.

$\vartheta=\begin{array}{llllll}193,8 & 198,1 & 193,7 & 193,2 & 193,5 & 193,5 \\ 193,9 & 193,4 & 193,6 & 194,5 & 193,5 & 194,0 \\ 193,7 & & & & & \end{array}$

Mittel $193,6^{0}$.

Zu den Mischungen von Methylalkohol und Aether wurde das zweite Destillat des Trommsdorff'schen Präparats benutzt.

I. 22,83 pC. Methylalkohol und 77,17 pC. Aether.

$$
\begin{array}{rrrrr}
\vartheta=200,2 & 200,8 & 199,9 & 200,7 & 200,4 \\
199,7 & 200,6 & 200,1 & 200,8 & 200,9 \\
& \text { Mittel } & 200,4^{0}, \\
\text { berechnet nach P a w l } 0 \text { w s k i } & 204,5^{\circ} .
\end{array}
$$

II. 54,33 pC. Aether und 45,67 pC. Alkohol.

$$
\begin{array}{rrrrrr}
\vartheta=211,9 & 212,1 & 211,7 & 213,0 & 211,8 & 212,7 \\
211,7 & 212,1 & 211,5 & 212,6 & 211,9 \\
& & & \text { Mittel } & 212,1^{\circ}, \\
& \text { berechnet nach P a w l e w s k i } & 215,7^{\circ} .
\end{array}
$$

III. 47,03 pC. Aether und 52,97 pC. Alkohol.

$$
\vartheta=\begin{array}{rlllll}
218,1 & 215,7 & 217,1 & 215,5 & 216,7 & 215,0 \\
216,1 & 216,2 & 216,7 & 215,3 & 216,7 & 215,2
\end{array}
$$

Mittel $216,2^{\circ}$,

berechnet nach Paw lewski 219,10.

II. Mischungen von Benzol und Aether.

Benzol, von Kahlbaum bezogen, wurde durch mehrmalige Krystallisation gereinigt und darauf in der Kälte mit 
kleinen Mengen von Natrium behandelt ${ }^{*}$ ). Siedepunkt $80,1^{0}$ beim Barometerstand $763,25 \mathrm{~mm}$.

$$
\begin{aligned}
& \vartheta=296,8 \quad 297,2 \quad 296,0 \quad 296,5 \quad 296,0 \\
& 297,0 \quad 295,8 \quad 296,2 \quad 295,8 \\
& \text { Mittel } 296,4^{0} \text {. }
\end{aligned}
$$

Nach Sajotschewski*) ist die kritische Temperatur $280,6^{0}$; nach Rams a y ${ }^{* *+}$ ) $291,5^{0}$; nach Young t) $288,5^{\circ}$.

Mischung I.

$$
\begin{array}{rrrrr}
85,77 & \text { pC. Aether } & \text { und } 14,23 & \text { pC. Benzol. } \\
\boldsymbol{7}=210,4 & 209,5 & 210,3 & 209,5 & 211,3 \\
209,2 & 210,0 & 209,4 & 210,5 & 209,4 \\
210,1 & 209,4 & & &
\end{array}
$$$$
\text { Mittel } 209,9^{\circ} \text {, }
$$

berechnet nach P awlewski 208, $1^{0}$.

Mischung II.

28,36 pC. Benzol und 71,64 pC. Aether.

\begin{tabular}{|c|c|c|c|c|}
\hline $9=232,2$ & 230,8 & 232,3 & 230,7 & 231,7 \\
\hline 230,5 & 232,1 & 231,0 & 281,7 & 230,9 \\
\hline 231,9 & 231,0 & & & \\
\hline
\end{tabular}

$$
\begin{aligned}
& \vartheta=225,1 \quad 225,9 \quad 224,7 \quad 225,8 \quad 222,8 \\
& 225,5 \quad 223,5 \quad 224,9 \quad 223,4 \quad 224,8 \\
& 223,3
\end{aligned}
$$

Mittel 224,50,

berechnet nach Pawlewski $223,7^{\circ}$.

Misehnng III.

$$
36,04 \text { pC. Benzol und 63,96 pC. Aether. }
$$

Mittel $231,4^{0}$,

berechnet nach Pawlewski $230,6^{\circ}$,

*) Nachdem die Versuche mit dem Benzol beendet waren, bemerkte ich, dafs Natrium namentlich in der Siedhitze auf Benzol einwirkt. Da die von mir gefundene kritische Temperatur höher liegt, als die von anderen beobachtete, so wird wohl eine geringe Zersetzung stattgefunden haben.

*) Beiblätter $\mathbf{8}, 741.1879$.

***) Proc. of Roy. Soc. 81, 194. 1881.

†) Journ. of Chem. Soc. 55, 507. 1889. 
Mischung IV.

$$
\begin{aligned}
& 52,74 \text { pC. Aether und 47,26 pC. Benzol. }
\end{aligned}
$$

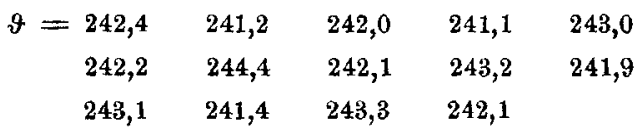

$$
\begin{aligned}
& \text { Mittel } 242,3^{\circ} \text {, } \\
& \text { berechnet nach } \mathrm{Pawlewski} \quad 242,1^{\circ} \text {. }
\end{aligned}
$$

III. Mischungen von Propylalkohol und Aether.

Das benutzte Präparat von Propylalkohol war von Tromms dorff bezogen. Nach dem Entwässern mittelst Kalk wurde es der fractionirten Destillation unterworfen. Siedepunkt $96, \mathbf{3}^{\circ}$ beim Barometerstand $759,05 \mathrm{~mm}$.

$$
\vartheta=\begin{array}{rlllll}
270,7 & 270,0 & 270,7 & 270,3 & 271,2 & 270,2 \\
270,6 & 269,7 & 270,8 & 270,1 & 271,2 & 270,2 \\
271,4 & 270,2 & & & &
\end{array}
$$

\section{Mittel 270,50.}

Die kritische Temperatur des Propylalkohols ist nach Ramsay und Young *) $263,7^{0}$; de Heen**) $261,0^{0}$; Nadejdine 254,1 bis $258,0^{\prime \prime}$.

Mischung 1 .

83,63 pC. Aether und 16,37 pC. Propylalkohol.

$$
\begin{array}{rrrrrr}
\vartheta=203,2 & 204,9 & 203,5 & 204,3 & 203,5 & 204,4 \\
203,7 & 204,5 & 203,7 & 204,1 & 203,6 \\
& & \text { Mittel } & 203,9^{\circ}, \\
\multicolumn{5}{c}{\text { berechnet nach } \mathbf{P} \text { aw lewski }} & 206,1^{\circ} .
\end{array}
$$

Mischung II.

80,45 pC. Aether und 19,55 pC. Propylalkohol.

$$
\vartheta=\begin{array}{llllll}
212,4 & 210,7 & 210,6 & 212,2 & 210,6 & 212,1 \\
211,8 & 210,2 & 211,4 & 210,4 & &
\end{array}
$$

Mittel 211,30,

$$
\text { berechnet nach P a w lew s k } 208,6^{\circ} \text {. }
$$

*) Transact. of Roy. Soc. 180, 156. 1889.

**) Recherches touchant la physique comparée, pag. 102.

***) Journ. der russ. phys,-chem. Ges. 14, 536. 1882. Beibl. 2, 678. 
$280 S c h m i d t$, ïber die kritischen Temperaturen

Mischung III.

66,21 pC. Aether und 33,79 pC. Propylalkohol.

$\boldsymbol{\vartheta}=\begin{array}{rlllll}222,2 & 220,4 & 221,9 & 220,7 & 222,0 & 220,3 \\ 221,8 & 220,4 & 222,0 & 220,3 & & \end{array}$

Mittel 221,20,

berechnet nach Pawlewski $219,6^{\circ}$.

Mischung IV.

64,84 pC. Aether und 35,16 pC. Propylalkohol.

$$
\begin{array}{rlllr}
\vartheta=225,4 & 223,8 & 225,2 & 224,2 & 225,1 \\
223,8 & 225,1 & 223,7 & 225,0 & 224,2 \\
\multicolumn{4}{c}{\text { Mittel } 224,5^{0},}
\end{array}
$$

berechnet nach Pawlewski 220, $6^{\circ}$.

IV. Mischungen von Aethylamin und Benzol.

Aethylamin, von Kahlbaum bezogen, wurde mittelst Kalk entwässert. Die kritische Temperatur war

$$
\begin{aligned}
& \vartheta=185,9 \quad 184,9 \quad 185,7 \quad 184,7 \\
& 185,6 \quad 184,9 \quad 185,7 \quad 185,0 \\
& 185,7 \quad 184,7 \quad 185,5 \quad 184,7
\end{aligned}
$$

Vincent und $\mathrm{Chappuis}$ *) haben die kritische Temperatur des Aethylamins zu $177,0^{\circ}$ bestimmt.

67,58 pC. Benzol und 32,42 pC. Aethylamin.

$$
\begin{aligned}
& \vartheta=258,2 \quad 259,2 \quad 258,0 \quad 257,6 \\
& 258,6 \quad 257,6 \quad 258,6 \quad 258,0
\end{aligned}
$$

berechnet nach Pawlewski 260,30.

V. Mischungen von Diäthylamin und Benzol.

Diäthylamin von Trommsdorff bezogen, wurde mittelst Kalk entwāssert.

*) Compt. rend. $108,379.1886$. 
Röhre I.

$$
\begin{array}{rlrr}
\vartheta=222,8 & 224,2 & 222,7 & 223,9 \\
222,6 & 223,7 & 222,9 & 222,4 \\
222,4 & 223,9 \\
c & \text { Mittel } & 223,1 .
\end{array}
$$

Röbre II.

$$
\begin{aligned}
& \vartheta=223,0 \quad 222,6 \quad 223,0 \quad 222,4 \\
& 224,0 \quad 222,4 \quad 224,2 \quad 223,0 \quad 222,6 \\
& \text { Mittel 223, } 0^{\circ} \text {. }
\end{aligned}
$$

Die kritische Temperatur des Diäthylamins ist demnach 223, $0^{\circ}$. Vincent und $\mathrm{Chappu \text {is }}{ }^{*}$ ) fanden $\vartheta=216,0^{\circ}$, Sa jo ts chewski*t) $220,0^{0}$, Kannegiefser $\left.222,8^{0 * *}\right)$.

Mischung I.

$$
\begin{array}{rrrrr}
73,19 & \text { pC. Diathylamin } & \text { und } & 26,81 & \text { pC. Benzol. } \\
\mathfrak{9}=240,8 & 240,1 & 242,4 & 241,1 & 242,6 \\
241,2 & 242,4 & 240,0 & 244,6 & 243,1 \\
\multicolumn{4}{c}{\text { Mittel }} & 241,8^{0},
\end{array}
$$$$
\text { berechnet nach Pawlewski } 242,6^{\circ} \text {. }
$$

Mischung II.

26,26 pC. Diëthylamin und 73,74 pC. Benzol.

$$
\begin{aligned}
& \vartheta=277,2 \quad 276,2 \quad 278,0 \quad 277,0 \quad 278,2 \\
& 277,0 \quad 278,5 \quad 277,2 \\
& \text { Mittel } 277,4^{0}, \\
& \text { berechnet nach } P a w l e w s k i \quad 276,4^{0} \text {. }
\end{aligned}
$$

Die kritische Temperatur der Mischung II stieg fortwährend; nach einer halben Stunde war sie circa $280,5^{\circ}$.

VI. Mischungen von Diäthylamin und Aether.

Zu den folgenden Versuchen wurde Aether, der von Kahlbaum bezogen und mit Phosphorsäureanhydrid und Natrium entwässert worden war, benutzt.

*) Compt. rend. $103,379.1886$.

**) Beiblätter 8, 741. 1879.

***) Journ. der russ. phys.-chem. Ges. 1 1, 304. Beiblatter 8, 808. 1884.

Annalen der Chemie 286. Bd. 
Röhre I.

$$
\begin{aligned}
& \vartheta=194,3 \quad 193,7 \quad 194,3 \quad 193,5 \quad 194,7 \quad 193,5 \\
& 194,2 \quad 193,3 \quad 194,7 \quad 192,7
\end{aligned}
$$

Röhre II.

$$
\vartheta=\begin{aligned}
& 194,7 \\
& 193,2
\end{aligned} \quad 192,9 \quad 194,7 \quad 193,2 \quad 194,3
$$

Mittel $193,8^{\circ}$.

Mischung I.

$$
\begin{aligned}
& 89,97 \text { pC. Aether und 10,03 pC. Aethylamin. }
\end{aligned}
$$

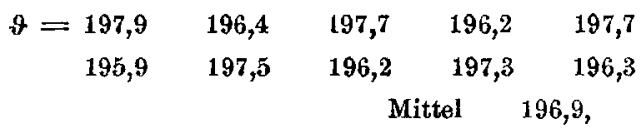$$
\text { berechnet nach } P \text { aw lewski } 196,7^{\circ} \text {. }
$$

Mischung II.

83,43 pC. Aether und 16,57 pC. Aethylamin.

$$
\begin{array}{rllll}
\vartheta=200,7 & 199,3 & 200,9 & 199,9 & 200,8 \\
199,5 & 200,7 & 199,3 & 201,1 & 199,7
\end{array}
$$

Mittel $200,2^{\circ}$,

berechnet nach Pawlewski $198,6^{\circ}$.

Mischung III.

69,51 pC. Aether und 30,49 pC. Diäthylamin.

$$
\begin{array}{rrrrrr}
\vartheta=204,5 & 208,1 & 204,7 & 202,2 & 204,9 & 202,7 \\
204,9 & 203,1 & 204,8 & 202,5 \\
& & \text { Mittel } & 203,7^{\circ}, \\
& \text { berechnet nach } & \text { Paw lew s k } & 202,7^{\circ} .
\end{array}
$$

Mischung IV.

53,56 pC. Aether und 46,44 pC. Aethylamin.

$$
\begin{array}{rrrrrr}
* 209,9 & 208,2 & 209,8 & 208,2 & 209,5 & 208,3 \\
209,4 & 208,1 & 209,7 & 207,7 & \\
& & & \text { Mittel } & 208,9^{\circ}, \\
& & & \text { berechnet } & 207,3^{\circ} .
\end{array}
$$

VII. Mischungen von Isopentan und Diäthylamin.

Das zu den folgenden Versuchen benutzte Pentan war von Trominsdorff bezogen. Nachdem es mit Phosphorsäureanhydrid entwässert worden war, wurde es der frac- 
tionirten Destillation unterworfen und der Theil, welcher zwischen 31 und $33^{\circ}$ beim Barometerstand 760,7 nim überging, besonders aufgefangen. Die kritische Temperatur dieses Theils war :

Röhre I.

$$
\begin{array}{rllll}
\vartheta=193,7 & 191,7 & 194,2 & 192,0 & 193,7 \\
191,7 & 193,5 & 192,1 & 193,4 & 192,1 \\
\multicolumn{4}{c}{\text { Mittel }} & \multicolumn{3}{c}{192,8^{\circ} .}
\end{array}
$$

Röhre II.

$$
\begin{aligned}
& \vartheta=194,7 \quad 192,1 \quad 194,7 \quad 192.5 \quad 194,5 \\
& 192,2 \quad 194,6 \quad 191,1 \\
& \text { Mittel } 193,3^{\circ} \text {. }
\end{aligned}
$$

Trotzdem die von mir bestimmte kritische Temperatur 193,0 $0^{\circ}$ des Isopentans gut mit der von Pawlewski*) gefundenen 194, $0^{\circ}$ übereinstimmt, wird mein Präparat wohl nicht rein gewesen sein, sondern eine beträchtliche Menge von Pentan enthalten haben **).

Mischung $\mathrm{I}$.

35,12 pC. Diäthylamin und 64,88 pC. Pentan.

$$
\begin{aligned}
& \vartheta=203,9 \quad 205,1 \quad 204,1 \quad 204,9 \\
& \text { Mittel } 204,5^{\circ} \text {, } \\
& \text { berechnet } 208,5^{\circ} \text {. }
\end{aligned}
$$

Mischung II.

65,99 pC. Diäthylamin und 34,01 pC. Pentan.

$$
\begin{aligned}
& \vartheta=213,5 \quad 211,7 \quad 213,2 \quad 211,7 \quad 213,1 \quad 211,4 \\
& 213,5 \quad 211,2 \quad 213,5 \quad 211,8
\end{aligned}
$$

*) Ber. d. deutsch. chem. Ges. 15, 2463. 1582.

**) Die Kohlenwasserstoffe lassen sich durch fractionirte Destillation nur schwierig von einander trennen. Perk in giebt den Siedepunkt eines von Schorlemmer mit der gröfsten Sorgfalt fractionirten Präparats von Isopentan zu 29 bis $32^{\circ}$, von Pentan zu 36 bis $36,5^{\circ}$ an. Journ. f. pr. Chem. [2] $\mathbf{3 1}, 488$. 
VIII. Mischungen von Propionsäure und Aether.

Propionsäure, von Kahlbaum bezogen, siedete nach dem Entwässern mit Phosphorsäureanhydrid constant bei $139,6^{\circ}$ beim Barometerstand $762,05 \mathrm{~mm}$.

$$
\vartheta=\begin{array}{rrrrr}
338,4 & 337,2 & 338,2 & 336,8 & 338,6 \\
336,0 & 338,8 & 336,5 & &
\end{array}
$$

Mittel $337,6^{\circ}$.

Pawlewski*) hat die kritische Temperatur der Propionsäure zu $339,9^{\circ}$ bestimmt.

24,74 pC. Propionsture und 75,26 pC. Aether.

$$
\begin{array}{rrrrr}
\vartheta=233,2 & 231,0 & 232,8 & 230,8 & 233,0 \\
230,5 & 233,0 & 230,8 & 232,6 & 231,2 \\
& & \text { Mittel } & 231,9^{\circ}, \\
& & \text { berechnet } & 229,4^{\circ} .
\end{array}
$$

IX. Mischungen von Essigsäuremethylester und Aether.

Essigsäuremethylester, von $\mathrm{Ka} \mathrm{h} \mathrm{l} \mathrm{b}$ a u m bezogen, wurde nach dem Trocknen mit Kupfersulfat der fractionirten Destillation unterworfen. Siedepunkt $57,1^{\circ}$ beim Barometerstand $756,20^{\circ}$.

Das Präparat wurde mehrmals mit Lackmus geprüf; während der Zeit, in der die nachstehenden Versuche angestellt wurden, trat keine Zersetzung ein.

Röhre I.

$$
\begin{aligned}
& \vartheta=235,4 \quad 236,1 \quad 235,2 \quad 236,0 \quad 235,4 \\
& 236,3 \quad 235,4 \quad 236,6 \quad 235,7 \quad 236,2 \\
& 235,4
\end{aligned}
$$

$$
\text { Mittel } 235,8^{\circ} \text {. }
$$

Röhre II.

$$
\begin{array}{rllll}
\vartheta=235,4 & 236,3 & 235,2 & 236,1 & 236,5 \\
235,6 & 236,4 & 235,6 & 236,6 & 235,4 \\
\multicolumn{4}{c}{\text { Mittel }} & 235,9^{\circ} .
\end{array}
$$

*) Ber. d. deutsch, chem. Ges. 15, 2403. 1882. 
Die kritische Temperatur des Aethers war $193,5^{\circ}$. Mischung $\mathrm{I}$.

51,19 pC. Essigsäuremethylester und 48,81 pC. Aether.

$$
\vartheta=\begin{array}{llllll}
212,4 & 211,2 & 212,3 & 211,4 & 212,4 & 211,3 \\
212,3 & 211,3 & 212,4
\end{array}
$$

Mischung II.

$$
\begin{array}{rr}
\text { Mittel } & 211,9^{\circ}, \\
\text { berechnet } & 215,1^{\circ} .
\end{array}
$$

58,96 pC. Essigsüuremethylester und 41,04 pC. Aether.

$$
\begin{aligned}
& \vartheta=217,5 \quad 216,9 \quad 217,4 \quad 216,3 \quad 217,6 \\
& 216,7 \quad 217,7
\end{aligned}
$$

Mischung IIr.

$$
\begin{aligned}
\text { Mittel } & 217,2^{\circ}, \\
\text { berechnet } & 218,4^{0} .
\end{aligned}
$$

13,61 pC. Essigsäuremethylester und 86,39 pC. Aether.

$$
\begin{array}{rrrrr}
\vartheta=198,6 & 197,7 & 198,4 & 197,9 & 198,6 \\
197,8 & 198,7 & 199,0 & 198,0 \\
& & \text { Mittel } & 198,4^{0}, \\
& & \text { berechnet } & 199,3^{\circ} .
\end{array}
$$

Mischung IV.

21,09 pC. Ester und 78,91 pC. Aether.

$$
\begin{array}{rrrrr}
\vartheta=203,2 & 201,2 & 202,8 & 201,2 & 202,2 \\
201,2 & 202,3 & 201,4 & 202,0 & 201,2 \\
& & \text { Mittel } & 201,9^{\circ} . \\
& & \text { berechnet } & 202,4^{\circ} .
\end{array}
$$

X. Mischungen von Tetrachlorkohlenstoff und Aether.

Tetrachlorkohlenstoff, von $\mathrm{Ka} \mathrm{h} \mathrm{l} \mathrm{b} \mathrm{a} \mathrm{u} \mathrm{m} \mathrm{bezogen,} \mathrm{wurde}$ nach dem Trocknen der fractionirten Destillation unterworfen. Siedepunkt $76,0^{\circ}$ beim Barometerstand $748,85 \mathrm{~mm}$.

Röhre I.

$$
\begin{aligned}
& \vartheta=285,0 \quad 284,4 \quad 284,9 \quad 284,4 \quad 285,2 \\
& 284,3 \quad 284,9 \quad 284,1 \quad 284,9 \quad 284,3 \\
& \text { Mittel } 284,6^{\circ} \text {. }
\end{aligned}
$$

Rohre II.

$$
\begin{array}{rlclr}
\vartheta=285,5 & 285,2 & 285,7 & 284,7 & 285,5 \\
284,8 & 285,5 & 284,7 & 285,7 & 284,8 \\
\multicolumn{4}{c}{\text { Mittel }} & 285,2^{\circ} .
\end{array}
$$


Der Inhalt der Röhre hatte sich etwas zersetzt.

Von den vielen Mischungen von Tetrachlorkohlenstoff und Aether, Tetrachlorkohlenstoff und Propylalkohol u. s. w., welche ich untersucht habe, theile ich nur zwei Beobachtungsreihen mit. Die Differenzen zwischen den beobachteten und berechneten Werthen waren in allen Fällen sehr grofs; da aber stets nachgewiesen werden konnte, dafs eine tiefgreifende Zersetzung stattgefunden, so können selbstverständlich diese Versuche zur Entscheidung der Frage, ob die Pawlewskische Formel für Flüssigkeitsgemische zulässig ist, oder nicht, nicht herangezogen werden.

72,79 pC. Tetrachlorkohlenstoff und 27,21 pC. Aether.

Röhre I.

$$
\begin{aligned}
& \vartheta=\begin{array}{rrrr}
248,6 & 249,6 & 247,8 & 249,8 \\
248,1 & 248,8 & 248,1 &
\end{array} \\
& \text { Mittel 248, } 8^{\circ} \text {, } \\
& \text { berechnet } 260,5^{\circ} \text {. }
\end{aligned}
$$

Röhre II.

$$
\begin{array}{rlrl}
\vartheta=253,0 & 252,0 & 253,3 & 251,8 \\
253,6 & 251,6 & \\
& \text { Mittel } & 252,5^{\circ} \\
& \text { berechnet } & 260,5^{\circ} .
\end{array}
$$

Nach der Untersuchung wurden beide Röhren geöffnet und mit Silbernitrat versetzt. Ein dicker weifser Niederschlag von Chlorsilber zeigte, dafs der Inhalt der Röhrchen zersetzt war.

Ich habe noch einige Mischungen mit Brom und Jodverbindungen untersucht, ebenso mit Schwefelkohlenstoff; in allen Fällen waren die Differenzen zwischen den beobachteten und den nach der Pawlewski'schen Formel berechneten kritischen. Temperaturen sebr grofs; stets erhielt ich beim Oeffnen der Röhre mit Silbernitrat resp. essigsaurem Blei einen Niederschlag, so dafs also sich stets Salzsäure resp. Schwefelwasser- 
stoff gebildet hat. Ich theile die erhaltenen Zahlen nicht mit, da die kritischen Temperaturen, soweit ich die Mischungen untersucht, niemals constant, sondern wie in dem oben genauer mitgetheilten Versuch, bei jedem Röhrchen verschieden waren.

In den folgenden Tabellen habe ich die Resultate meiner Experimente zusammengestellt. Mitaufgenommen habe ich noch die Versuche von Straufs*) über Aethylalkohol und Aether, sowie die von B. Galitzine ${ }^{* *}$ ) über Aceton und Aether.

\begin{tabular}{c|c|c|c|c}
\hline \multicolumn{2}{c|}{ Procentgehalt } & \multicolumn{2}{c|}{ Kritische Temperatur } & Differenz \\
\cline { 1 - 4 } $\begin{array}{c}\text { Methyl- } \\
\text { alkohol }\end{array}$ & Aethyläther & Beobachtet & $\begin{array}{c}\text { Nach Pawl. } \\
\text { berechnet }\end{array}$ & \\
\hline 0 & 100 & 193,5 & - & - \\
22,83 & 77,17 & 200,4 & 204,5 & $-4,1$ \\
45,67 & 54,33 & 212,1 & 215,7 & $-3,6$ \\
52,97 & 47,03 & 216,2 & 219,1 & $-2,9$ \\
100 & 0 & 241,9 & - & -
\end{tabular}

\begin{tabular}{c|c|c|c|c}
\hline \multicolumn{2}{c|}{ Procentgehalt } & \multicolumn{2}{c|}{ Kritische Temperatur } & \multirow{2}{*}{ Differenz } \\
\cline { 1 - 4 } Aethylalkohol & Aether & Beobachtet & Berechnet & \\
\hline 0 & 100 & 195,5 & - & - \\
15,2 & 84,8 & 202,8 & 202,3 & $+0,5$ \\
27,8 & 72,2 & 208,8 & 208,0 & $+0,8$ \\
52,8 & 47,2 & 218,8 & 219,3 & $-0,5$ \\
72,7 & 27,3 & 227,5 & 228,3 & $-0,8$ \\
83,9 & 16,1 & 233,9 & 233,3 & $+0,6$ \\
96,5 & 3,5 & 239,9 & 239,0 & $+0,9$ \\
100,0 & 0 & 240,6 & - & -
\end{tabular}

*) Beibl. 6, 282.

**) Wied. Ann: 41, 624 . 


\begin{tabular}{|c|c|c|c|c|}
\hline \multicolumn{2}{|c|}{ Procentgehalt } & \multicolumn{2}{|c|}{ Kritische Temperatur } & \multirow{2}{*}{ Differenz } \\
\hline Propylalkohol & Aether & Beobachtet & Berechnet & \\
\hline 100 & 0 & 270,5 & - & 一 \\
\hline 35,16 & 64,84 & 224,5 & 220,6 & $+3,9$ \\
\hline 33,79 & 66,21 & 221,2 & 219,6 & $+1,6$ \\
\hline 19,55 & 80,45 & 211,3 & 208,6 & $+2,7$ \\
\hline 16,37 & 83,63 & 203,9 & 206,1 & $-2,2$ \\
\hline 0 & 100,0 & 193,5 & - & - \\
\hline
\end{tabular}

\begin{tabular}{c|c|c|c|c}
\hline \multicolumn{2}{c|}{ Procentgehalt } & \multicolumn{2}{c|}{ Kritische Temperatur } & \multirow{2}{*}{ Differenz } \\
\cline { 1 - 4 } Benzol & Aether & Beobachtet & Berechnet & \\
\hline 100 & 0 & 296,4 & - & - \\
47,26 & 52,74 & 242,3 & 242,1 & $+0,2$ \\
36,04 & 63,96 & 231,4 & 230,6 & $+0,8$ \\
28,36 & 71,64 & 224,5 & 223,7 & $+0,8$ \\
14,23 & 85,77 & 209,9 & 208,1 & $+1,8$ \\
0 & 100,0 & 193,5 & - & -
\end{tabular}

\begin{tabular}{c|c|c|c|c}
\hline \hline \multicolumn{2}{c|}{ Procentgehalt } & \multicolumn{2}{|c|}{ Kritische Temperatur } & \multirow{2}{*}{ Differenz } \\
\hline Aethylamin & Benzol & Beobachtet & Berechnet & \\
\hline 100,0 & 0 & 185,2 & - & - \\
32,42 & 67,58 & 258,3 & 260,3 & $-2,0$ \\
0 & 100,0 & 296,4 & - & -
\end{tabular}

\begin{tabular}{c|c|c|c|c}
\hline \multicolumn{2}{c|}{ Procentgehalt } & \multicolumn{2}{c|}{ Kritische Temperatur } & \multirow{2}{*}{ Differenz } \\
\cline { 3 - 5 } Diathylamin & Benzol & Beobachtet & Berechnet & \\
\hline 100,0 & 0 & 223,0 & - & - \\
73,19 & 26,81 & 241,8 & 242,6 & $-0,8$ \\
26,26 & 73,74 & 277,4 & 276,4 & $+1,0$ \\
0 & 100 & 296,4 & - & -
\end{tabular}


von Fliissigkeitsgemischen.

\begin{tabular}{|c|c|c|c|c|}
\hline \multicolumn{2}{|c|}{ Procentgehalt } & \multicolumn{2}{|c|}{ Kritische Temperatur } & \multirow{2}{*}{ Differenz } \\
\hline Diäthylamin & Aether & Beobachtet & Berechnet & \\
\hline 100 & 0 & 222,9 & 一 & - \\
\hline 46,44 & 53,56 & 208,9 & 207,3 & $+1,6$ \\
\hline 30,49 & 69,51 & 203,7 & 202,7 & $+1,0$ \\
\hline 16,57 & 83,43 & 200,2 & 198,6 & $+1,6$ \\
\hline 10,03 & 89,97 & 196,9 & 196,7 & $+0,2$ \\
\hline 0 & 100,0 & 193,8 & - & - \\
\hline \multicolumn{2}{|c|}{ Procentgehalt } & \multicolumn{2}{|c|}{ Kritische 'Temperatur } & \multirow{2}{*}{ Differenz } \\
\hline Diathylamin & Isopentan & Beobachtet & Berechnet & \\
\hline 100 & 0 & 222,9 & - & - \\
\hline 65,99 & 34,01 & 212,5 & 212,7 & $-0,2$ \\
\hline 35,12 & 64,88 & 204,5 & 203,5 & $-1,0$ \\
\hline 0 & 100 & 193,0 & - & 一 \\
\hline
\end{tabular}

\begin{tabular}{c|c|c|c|c}
\hline \multicolumn{2}{c|}{ Procentgehalt } & \multicolumn{2}{c|}{ Kritische Temperatur } & \multirow{2}{*}{ Differenz } \\
\cline { 1 - 4 } Propionsëure & Aether & Beobachtet & Berechnet & \\
\hline \multirow{2}{*}{100} & 0 & 337,6 & - & - \\
24,74 & 75,26 & 231,9 & 229,4 & $-2,5$ \\
0 & 100 & 193,8 & - & -
\end{tabular}

\begin{tabular}{c|c|c|c|c}
\hline \multicolumn{2}{c|}{ Procentgehalt } & \multicolumn{2}{c|}{ Kritische Temperatur } & \multirow{2}{*}{ Differenz } \\
\cline { 1 - 4 } $\begin{array}{c}\text { Essigsäure- } \\
\text { methylester }\end{array}$ & Aether & Beobachtet & Berechnet & \\
\hline 100 & 0 & 235,8 & - & - \\
58,96 & 41,04 & 217,2 & 218,4 & $-1,2$ \\
51,19 & 48,81 & 211,9 & 215,1 & $-3,2$ \\
21,09 & 78,91 & 201,9 & 202,4 & $-0,5$ \\
13,61 & 86,39 & 198,4 & 199,3 & $-0,9$ \\
0 & 100 & 193,5 & - & -
\end{tabular}




\begin{tabular}{c|c|c|c|c}
\hline \multicolumn{2}{c|}{ Procentgehalt } & \multicolumn{2}{c|}{ Kritische Temperatur } & \multirow{2}{*}{ Differenz } \\
\cline { 1 - 3 } Aethyläther & Aceton & Beobachtet & Berechnet & \\
\hline 0 & 100 & 234,4 & - & - \\
6,6 & 93,4 & 230,1 & 231,6 & $-1,5$ \\
14,1 & 85,9 & 227,3 & 228,4 & $-1,1$ \\
30,7 & 69,3 & 218,7 & 221,3 & $-2,6$ \\
100,0 & 0 & 191,8 & - & -
\end{tabular}

Die von mir erhaltenen kritischen Temperaturen der Gemische von Tetrachlorkohlenstoff und Aether, sowie B. Galitzine's Versuche über Schwefelkohlenstoff und Aether habe ich wegen der vorhin mitgetheilten Gründe nicht berücksichtigt.

Die Differenzen zwischen den beobachteten und den nach der Pawlewski'schen Formel berechneten Werthen sind nicht grofs, nämlich im Maximum $3,9^{\circ} \mathrm{C}$. Die beobachteten kritischen Temperaturen sind theils höher, theils niedriger als die berechneten, rühren also wahrscheinlich von Beobachtungsfehlern her. Bei den meisten Beobachtungsreihen sind allerdings die Differenzen entweder stets positiv oder stets negativ; diese Beständigkeit der Unterschiede scheint dafür za sprechen, dafs die Pawle wski'sche Formel die kritischen Temperaturen von Flüssigkeitsgemischen nicht ganz genau wiedergiebt, doch können die Abweichungen auch durch geringe Zersetzungen der angewandten Substanzen erklärt werden. Bei den hohen Temperaturen, denen die Flüssigkeiten ausgesetzt werden müssen, wird wohl stets eine, wenn auch nur geringe Zersetzung eintreten, und da bei jedem Versuch wahrscheinlich dieselben Producte entstehen, so werden auch die beobachteten kritischen Temperaturen entweder stets höher oder stets tiefer liegen als die berechneten.

Bekanntlich zeigt die gemeinsame Dampfspannung von Flüssigkeiten sehr verwickelte Verhältnisse, die von der gegen- 
seitigen Einwirkung der Flüssigkeiten auf einander und auf die entstandenen Dämpfe herrühren. Bei Flüssigkeiten, welche in allen Verhältnissen in einander löslich sind (hierzu gehören alle Flüssigkeiten oberhalb der kritischen Lösungstemperatur), erreicht die gemeinsame Dampfspannung niemals die Summe der Einzelspannungen mehr, sie braucht auch nicht zwischen dieser und der höheren oder der niederen zu liegen, sondern sie kann sogar unter den Werth herabsinken, welchen die kleinere Einzelspannung hat. Dafs ähnliche Verhältnisse beim kritischen Druck eintreten würden, war zu erwarten. Da die Dampfspannungen von Flüssigkeitsgemischen besonders eingehend von Ko now alo $\mathrm{w}^{*}$ ) an Gemengen von Wasser mit den Fettalkoholen und Feltsäuren studirt worden sind, so suchte ich die kritischen Temperaturen von diesen Mischungen zu bestimmen. Die Röhren wurden jedoch so stark angegriffen, dafs ich von diesen Experimenten abstehen mufste $* *$ ).

B. Galitzin $e^{* * *}$ ) hat aus der Clausius'schen $\mathrm{Zu}-$ standsgleichung für die durch ein indifferentes Gas abgeänderte kritische Temperutur folgende Formel abgeleitet :

$$
\mathrm{T}_{2}^{12}=\frac{8\left(\mathrm{a}_{2}+\mathrm{a}_{21}\right)}{27\left(\mathrm{~b}_{2}+\mathrm{b}_{21}+\beta\right) \mathbf{R}_{2}} .
$$

Eine Prüfung der Gleichung ist leider nicht möglich, weil die Constanten $b$ und $\beta$ nicht getrennt bekannt sind. Bei passender Combination der in der Formel vorkommenden Gröfsen läfst sich die Möglichkeit solcher Fälle erkennen, in

*) Wied, Ann, 14, 34 und 219.

**) Aus Mischungen von Wasser und Alkohol hat Strauโs, Beibl. 7, 676, die kritische Temperatur des Wassers zu $370^{\circ} \mathrm{C}$. bestimmt. Mir gelang es ebensowenig wie Pawlewski, Ber. d. deutsch. chem. Ges. 15, 462, die kritische Temperatur von Gemengen von Alkohol und Wasser zu bestimmen, da die Versuchsrohre stets zersetzt wurden.

***) Wied. Ann. 41, 799 . 
denen die kritische Temperatur $\mathrm{T}_{2}^{1}$ selbst aufserhalb $\mathrm{T}_{1}$ und $T_{2}$ der Bestandtheile liegen kann. Bis jetzt ist jedoch ein sicheres Beispiel hierfür noch nicht bekannt.

Bekanntlich hat $\mathrm{L}$ andolt ${ }^{*}$ ) nachgewiesen, dafs die Brechungscoëfficienten von Gemengen zu denen der Bestandtheile in der Beziehung stehen

$$
p \cdot \frac{n-1}{d}=p_{1} \frac{n_{1}-1}{d_{1}}+p_{2} \frac{n_{2}-1}{d_{2}}+p_{3} \frac{n_{3}-1}{d_{s}}+\ldots
$$

wo $p, n$ und $d$ Gewicht, Brechungscoëfficient und Dichte des Gemenges $p_{1}, p_{2} \ldots n_{1}, n_{2} \ldots d_{1}, d_{z}$ die entsprechenden Werthe der Bestandtheile bedeuten. Die Formel ist dieselbe wie die von Pawlewski.

Eberswalde. Laboratorium des Herrn Prof. Remelé. Juli 1891.

\section{Glycocoll und Derivate;}

nach Versuchen von B. Goldberg, Ph. Kunz und nach eigenen Versuchen, mitgetheilt von $K$. Kraut.

(Eingelaufen den 15. August 1891.)

Meine letzte, vor 25 Jahren veröffentlichte Untersuchung über Derivate des Glycocolls **) mufste früher, als ich beabsichtigt hatte, abgebrochen werden, weil das Ausgangsmaterial, über das ich verfügte, verbraucht war. Bei Wiederaufnahme derselben war ich bestrebt, mit Umgehung der schwierig in ausreichender Menge zu beschaffenden Hippursäure, eine aus-

*) Pogg. Ann. 123, 595. 1864.

**) Diese Annalen 182, 172. 